\title{
Comparison of aspiration protection by extraglottic airways in an anatomically-correct regurgitation model
}

DEPARTMENT OF ANAESTHESIA \& PERIOPERATIVE MEDICINE UNIVERSITY OF CAPE TOWN
Ross Hofmeyr ${ }^{1}$, Donald Miller ${ }^{2}$

'University of Cape Town, Department of Anaesthesia and Perioperative Medicine, Cape Town, South Africa 2Guy's \& St Thomas'NHS Foundation Trust, Department of Anaesthesiology, London, United Kingdom

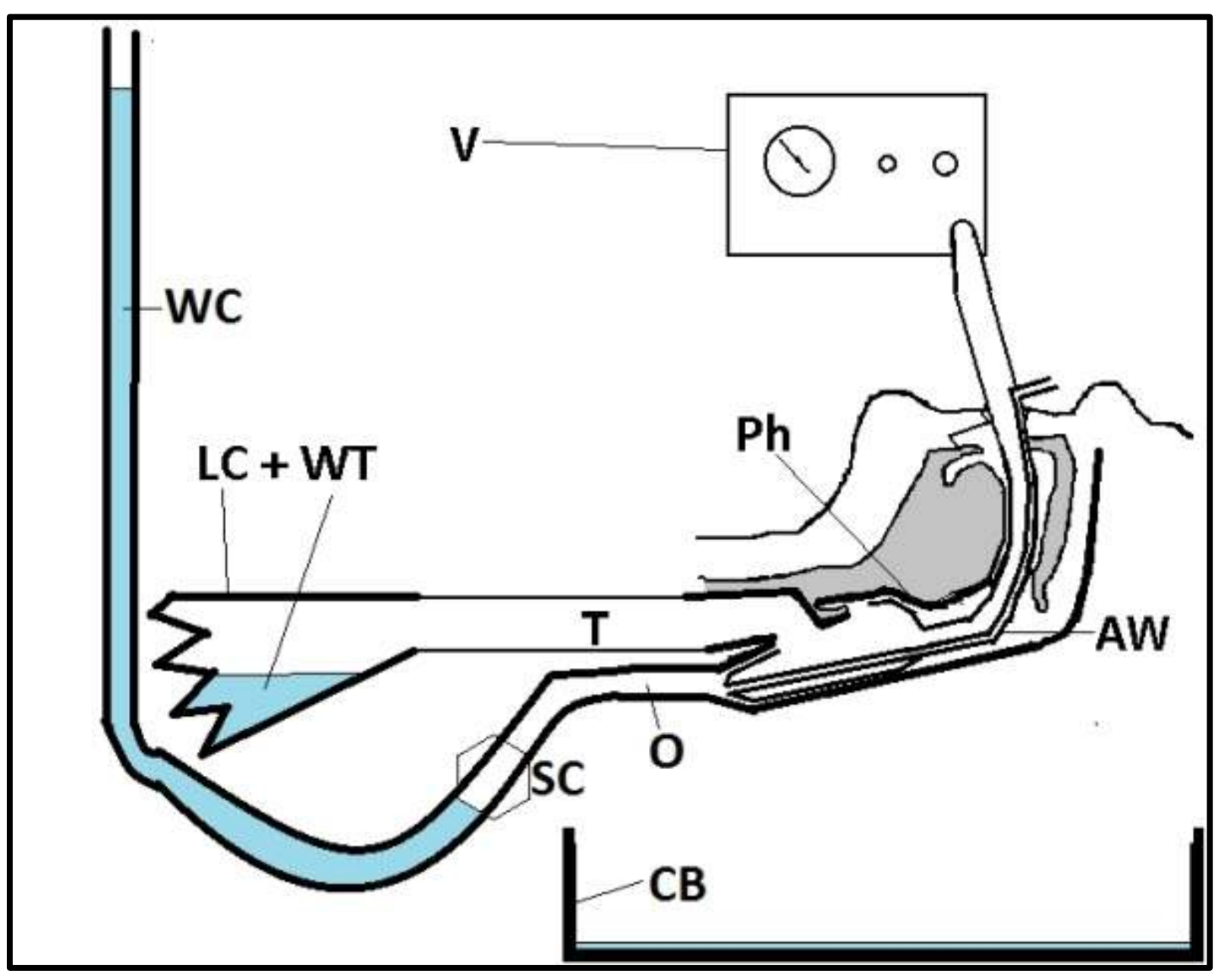

\section{Background and Goal}

The most common cause of airway morbidity/mortality during anaesthesia or resuscitation is aspiration of gastric contents.

Even the traditional gold standard (a cuffed endotracheal tube) does not guarantee protection. Extraglottic airway device (EAD; supraglottic airway; SGA) use has steadily increased, playing vital roles in routine and difficult airway management. However, EADs may be associated with increased aspiration, and employ many design features to reduce risk. In vivo heterogeneity has led to limited literature robustly comparing devices. Previous studies have used small numbers or single devices using dye or cadaveric models. ${ }^{1,2}$ We developed a laboratory model to allow repeated, accurate and direct testing.

\section{Materials and Methods}

An airway model $(\mathrm{Ph})$ was created using latex moulds of cadavers in combination with three-dimensional computed tomography scans of normal individuals. An elastomer with compliance similar to human tissue was selected. The trachea $(T)$ was connected to a test lung $(L C)$, and oesophagus $(O)$ via a stopcock (SC) to a water column (WC), allowing simulated passive regurgitation. A leak-compensating pressure-controlled ventilator (V) delivered intermittent positive pressure ventilation with and without positive end-expiratory pressure (PEEP). Water (WT) entering the test lung during each iteration was measured. Thirteen SGA/EAD configurations (AW) were tested, with 20 iterations each ( 10 with PEEP).

\section{Results and Discussion}

The complete data set and summary descriptive statistics are shown in the figure and table. Devices could be grouped into three categories: those providing complete (or near-complete) protection, partial protection, and no protection. Those designed with features which provide aspiration protection (e.g. drainage channels) outperformed basic devices when confronted with

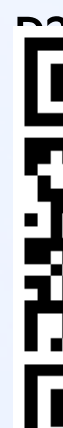

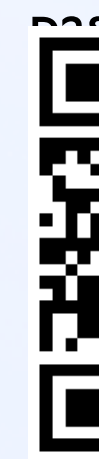

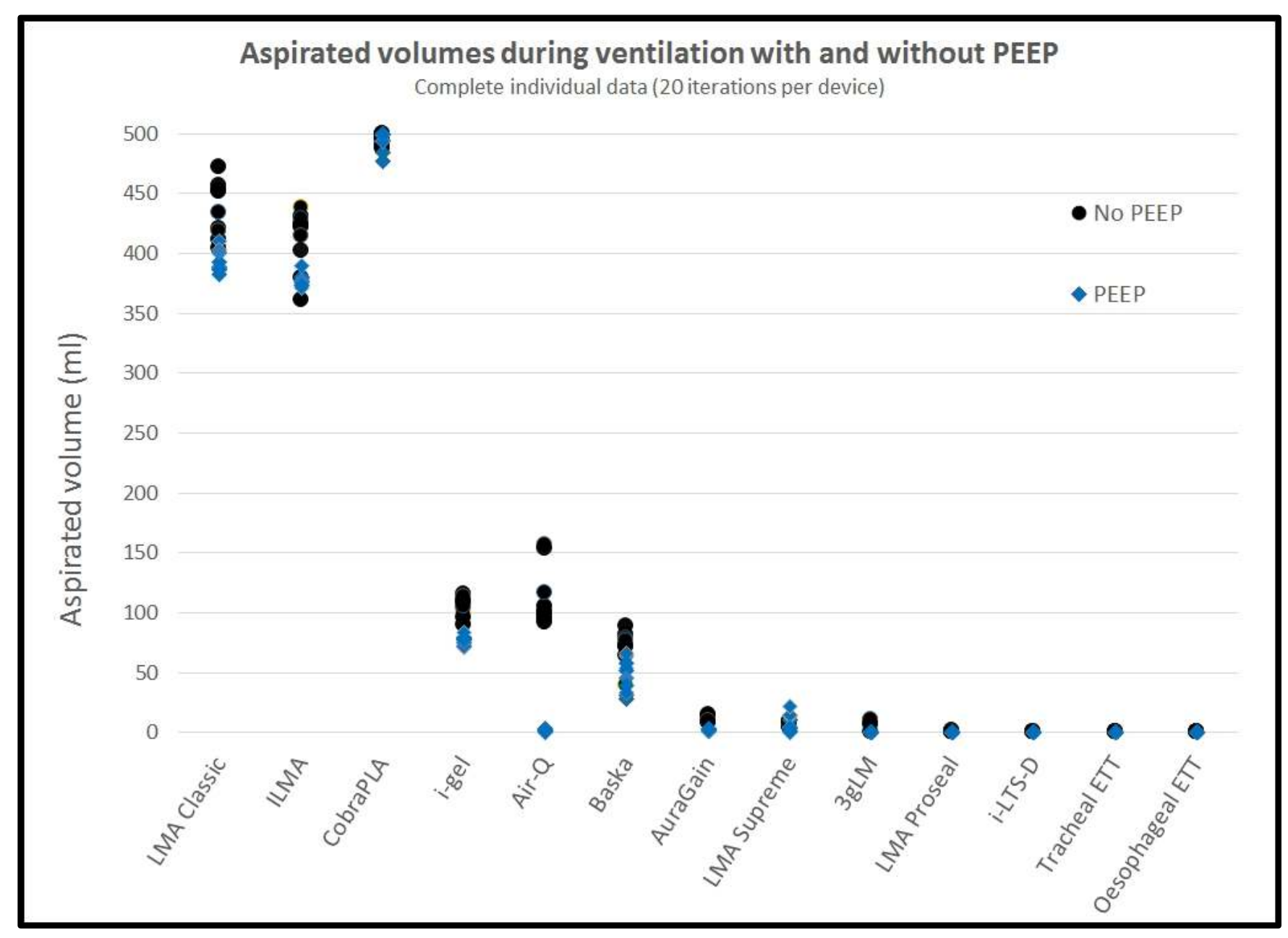

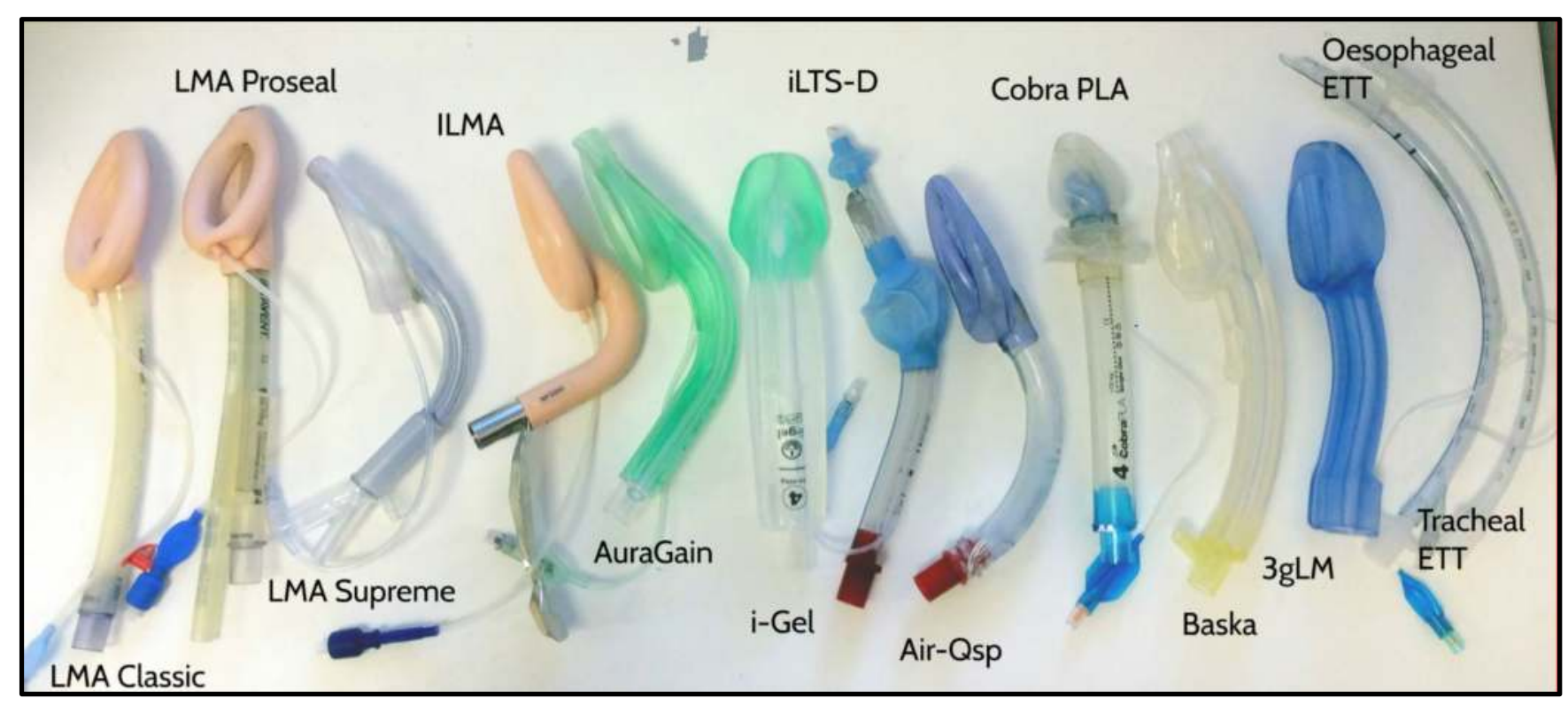

\begin{tabular}{|c|c|c|c|c|c|c|c|c|}
\hline \multirow[t]{2}{*}{ Device } & Mean (SD) & Min & nedian & Max & Mean (SD) & Min & Median & Max \\
\hline & \multicolumn{4}{|c|}{$\begin{array}{l}\text { Passive regurgitation } \\
\end{array}$} & \multicolumn{4}{|c|}{ Passive regurgitation with PEEP } \\
\hline LMA Classic & $437(23)$ & 404 & 443 & 472 & $394(9)$ & 382 & 391 & 410 \\
\hline ILMA & $412(25)$ & 361 & 422 & 438 & $377(5)$ & 371 & 376 & 390 \\
\hline CobraPLA & $496(5)$ & 487 & 497 & 505 & $494(8)$ & 477 & 499 & 500 \\
\hline i-gel & $105(8)$ & 90 & 107 & 115 & $76(4)$ & 71 & 76 & 83 \\
\hline Air-Q & $111(24)$ & 92 & 101 & 156 & $1(1))$ & 0 & 1 & 3 \\
\hline Baska & $72(13)$ & 39 & 75 & 89 & 47 (14) & 28 & 49 & 66 \\
\hline AuraGain & $10(2)$ & 8 & 10 & 15 & $2(2)$ & 1 & 2 & 3 \\
\hline LMA Supreme & $7(2)$ & 4 & 7 & 10 & $9(7)$ & 0 & 8 & 22 \\
\hline 3gLM & 7 (3) & 0 & 9 & 11 & $0(0)$ & 0 & 0 & 0 \\
\hline LMA Proseal & $0(0)$ & 0 & 0 & 1 & $0(0)$ & 0 & 0 & 0 \\
\hline i-LTS-D & $0(0)$ & 0 & 0 & 0 & $0(0)$ & 0 & 0 & 0 \\
\hline ETT Trachea & $0(0)$ & 0 & 0 & 0 & $0(0)$ & 0 & 0 & 0 \\
\hline ETT oesophagus & $0(0)$ & 0 & 0 & 0 & $0(0)$ & 0 & 0 & 0 \\
\hline
\end{tabular}

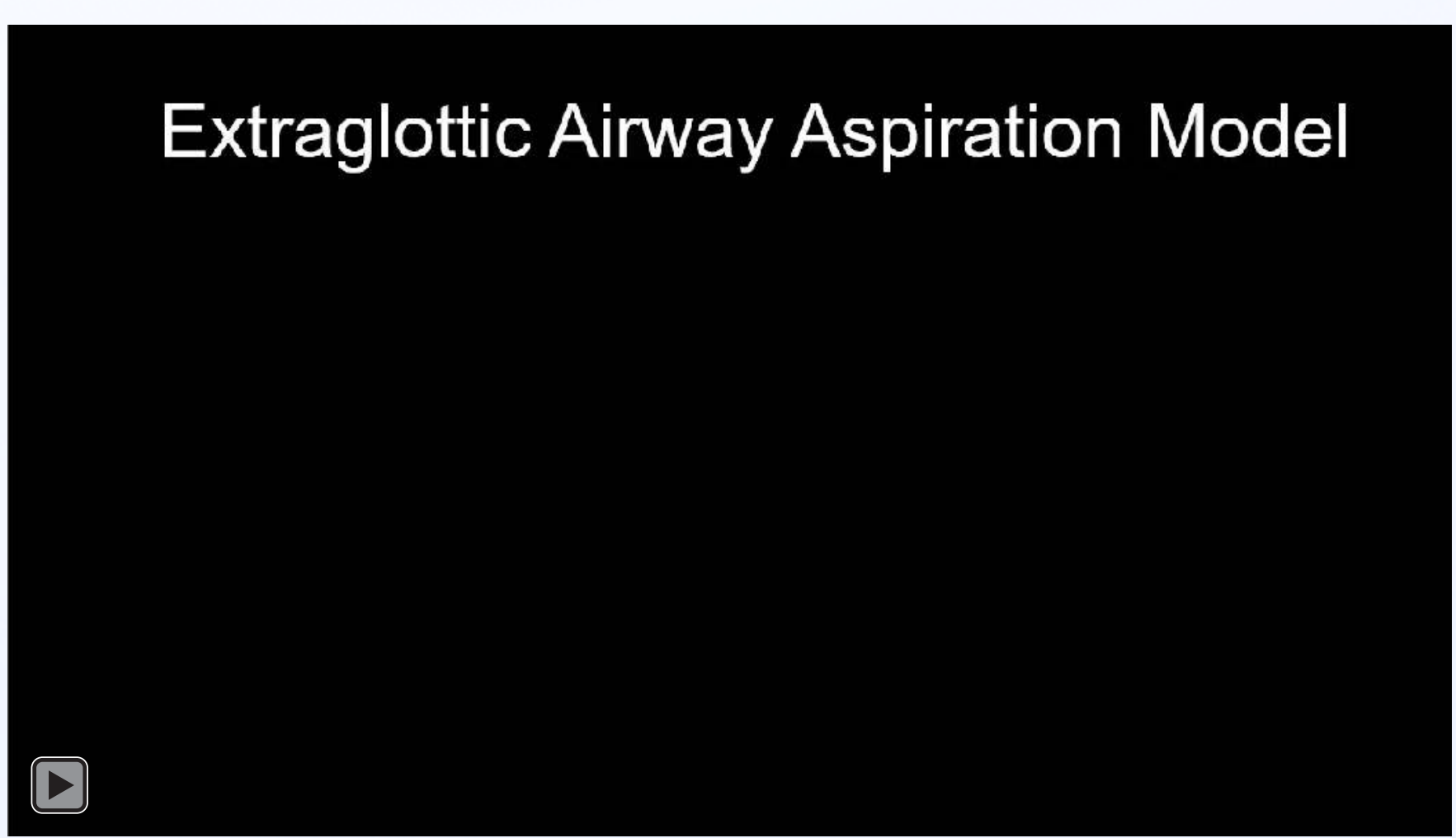

Video demonstration of aspiration model in use, showing excellent seal but no aspiration protection offered by Cobra PLA, and complete protection by i-LTS-D. 